\title{
High serum and epidermal extracellular levels of galectin-7
}

in atopic dermatitis

Takatsune Umayahara1), Masahiro Aoshima1), Manami Iwasaki1), Tsuyoshi Yatagai1),

Jun-ichi Sakabe ${ }^{2)}$, Yoshiki Tokura1), Takatoshi Shimauchi1)

1) Department of Dermatology, Hamamatsu University School of Medicine, Japan

2) Institute of Medical Biology, Agency for Science, Technology and Research (A*STAR), Singapore

Conflict of Interest: None

\section{Background}

- Atopic dermatitis (AD) patients show an impaired barrier function with Th2 dominant skin inflammation - Galectin-7 is specifically developed in epidermal keratinocytes

(Sato S et al. Trends Glycosci. Glycotechnol. 2002) - Galectin-7 was highly expressed in the cornified layer of AD patients (Sakabe J et al. J Allergy Clin Immunol. 2014)
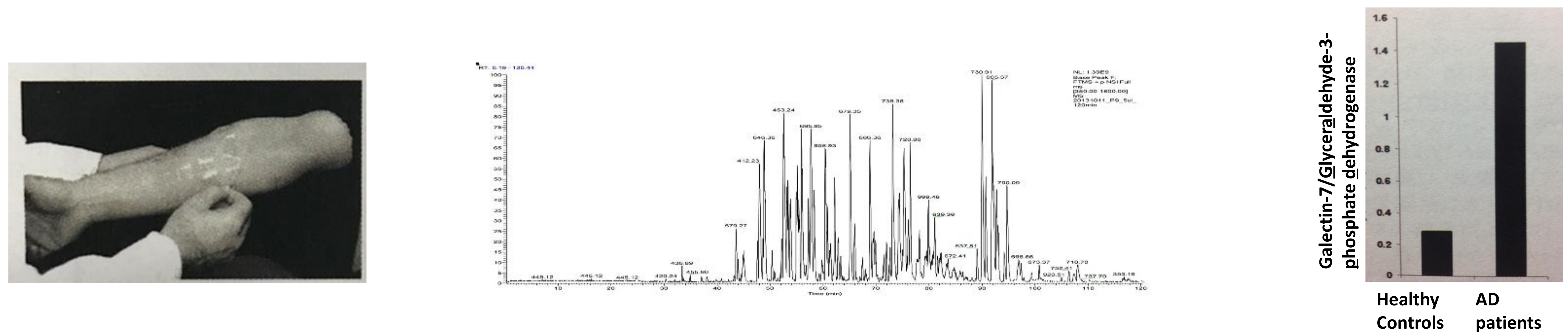

(Niiyama S et al. Int J Cosmet Sci, 2016)

\section{Objective}

$\checkmark$ To investigate the relationship between serum Galectin- 7 levels and AD-associated markers

$\checkmark$ To evaluate the Galectin-7 expression pattern in lesional skin in AD

$\checkmark$ To clarify the regulation of Galectin-7 production in epidermal keratinocytes by Th1, Th2, and Th17/22 cytokines

\section{Results}

Fig 1 A serum Galectin-7 levels correlates with both

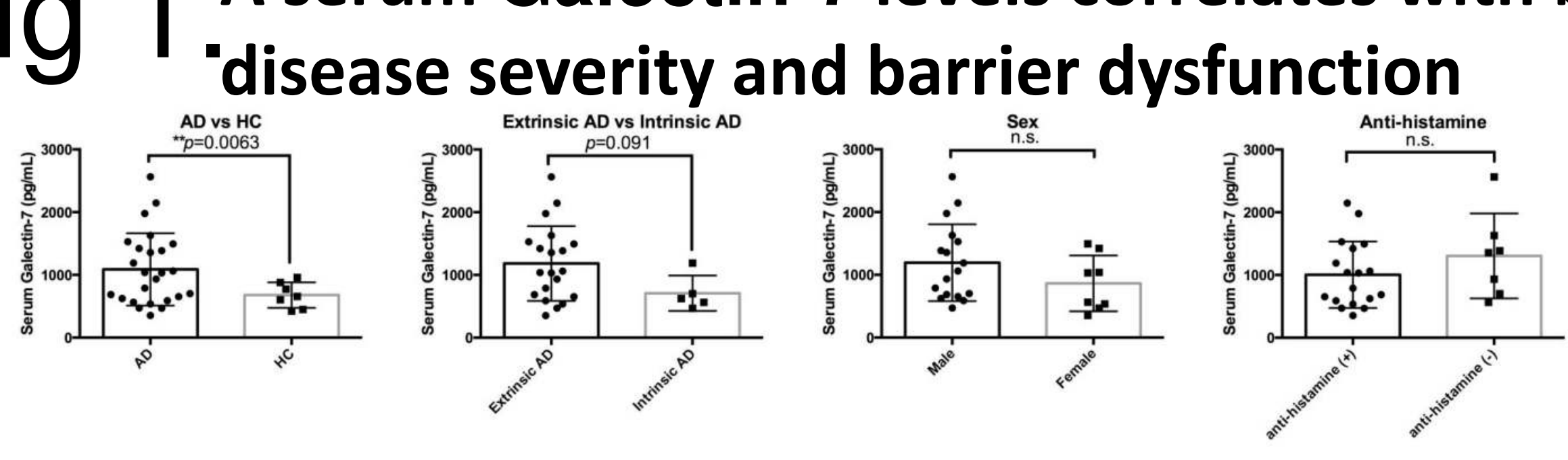

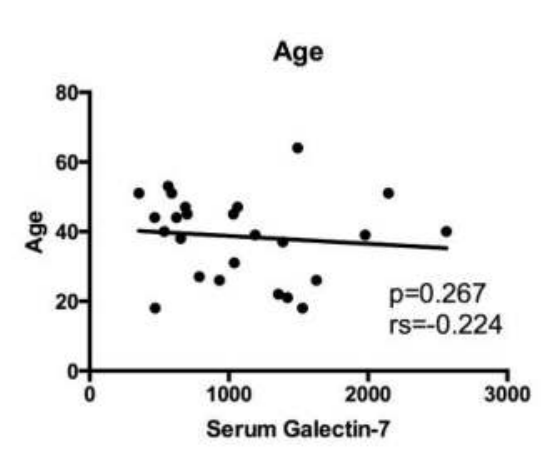
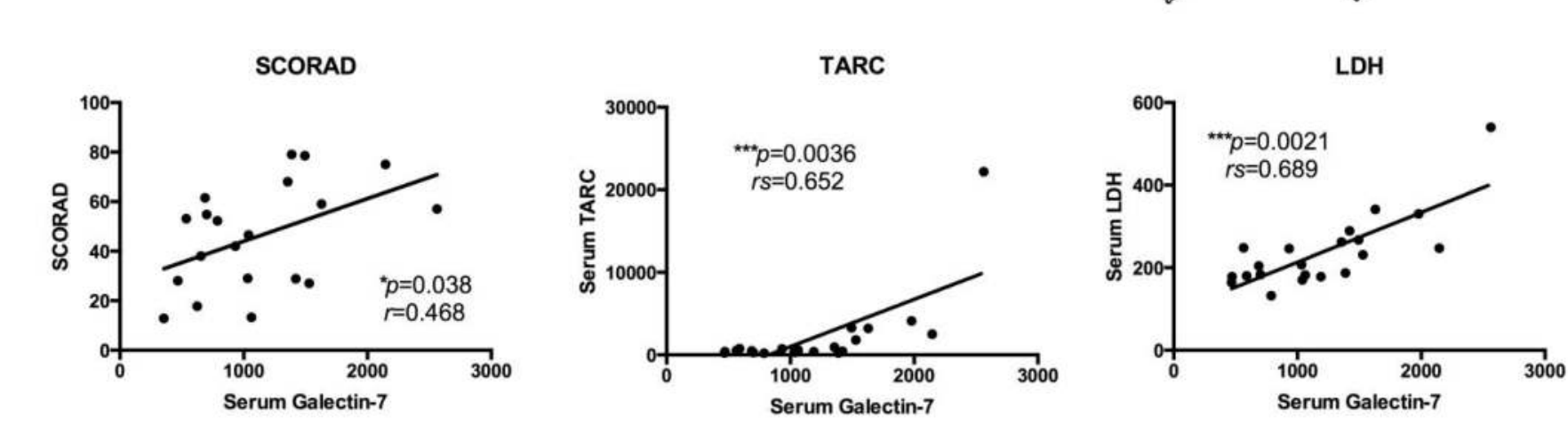

.
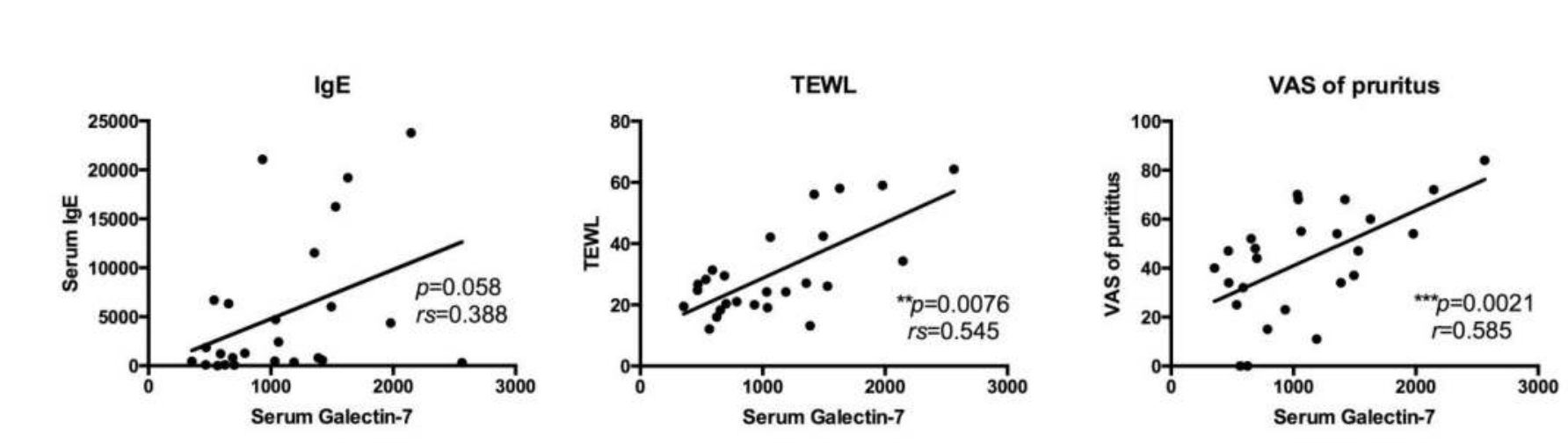

25 Japanese $A D$ patients and 7 healthy controls were enrolled in this study.

A serum galetin-7 level was significantly increased in $A D$ patients compared to healthy controls. Among total AD patients, a serum galectin-7 positively correlated with SCORAD, (CCL17/)TARC, LDH, \% eosinophils, TEWL, and pruritus.

※AD patients were also divided into extrinsic types(high IgE levels) and intrinsic (normal IgE levels) ones.
Fig 2 Galectin-7 is expressed in the intercellular space
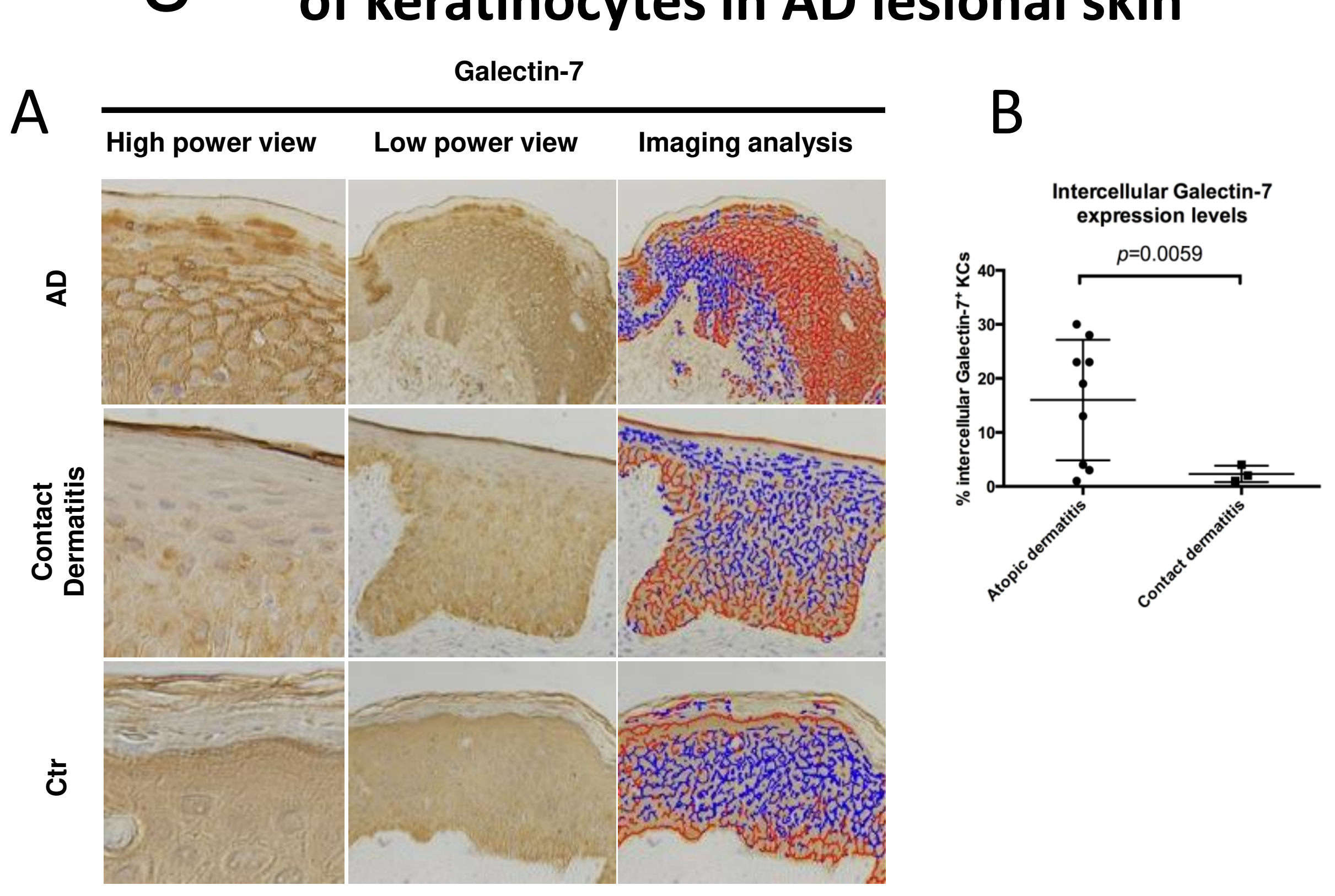

Immunohistochemical staining showed that, in both contact dermatitis and normal controls, galectin-7 was expressed in the cytoplasm of the prickle layer of keratinocytes. On the other hand, in AD lesional skin, the galectin-7 was positively stained in the intercellular space of epidermal keratinocytes and was also

accumulated in the cornified layer (2-A).

Computer imaging analysis also revealed that the intensity of galectin-7 of intercellar space of keratinocytes was significantly higher in AD patients than those in contact dermatitis patients (2-B).
Fig 3. IL-4/IL-13 increase Galectin-7 production from

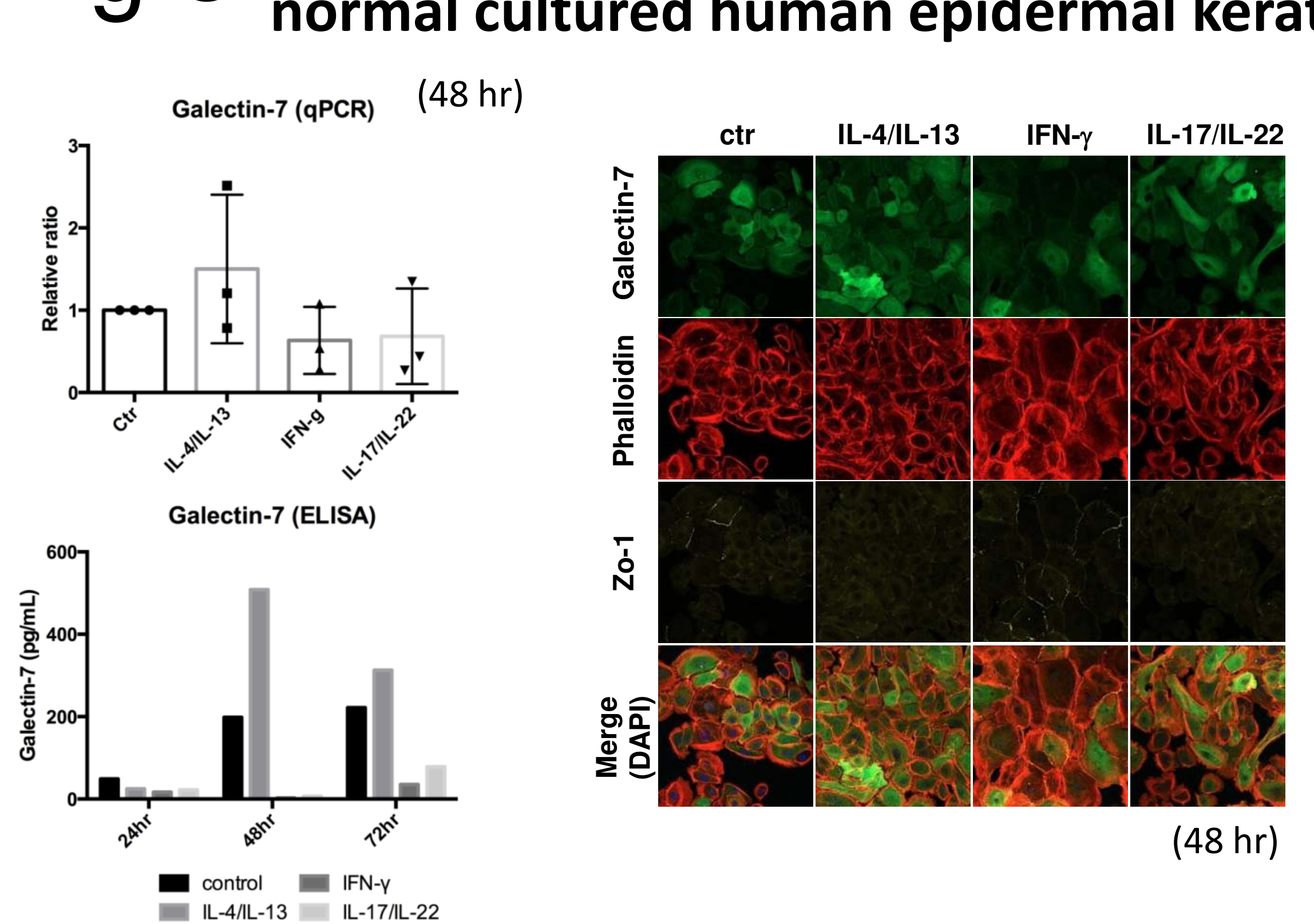

To quantify the galectin-7 production, we cultured monolayer normal human epidermal keratinocytes (NHEK) with or without IL-4/IL-13, IFN- $\mu$, or IL-17/IL22, and analyzed by Quantitative PCR(qPCR), ELISA, and confocal microscopy.

IL-4/IL-13 treatment increased the galectin-7 production from keratinocytes at $48 \mathrm{hr}$ compared to other cytokines as shown in qPCR, ELISA and confocal imaging.

\section{Fig 4 Galectin-7 is extracellularly released from}

ig 4. epidermal keratinocytes in 3D cultured keratinocytes by IL-4/IL-13
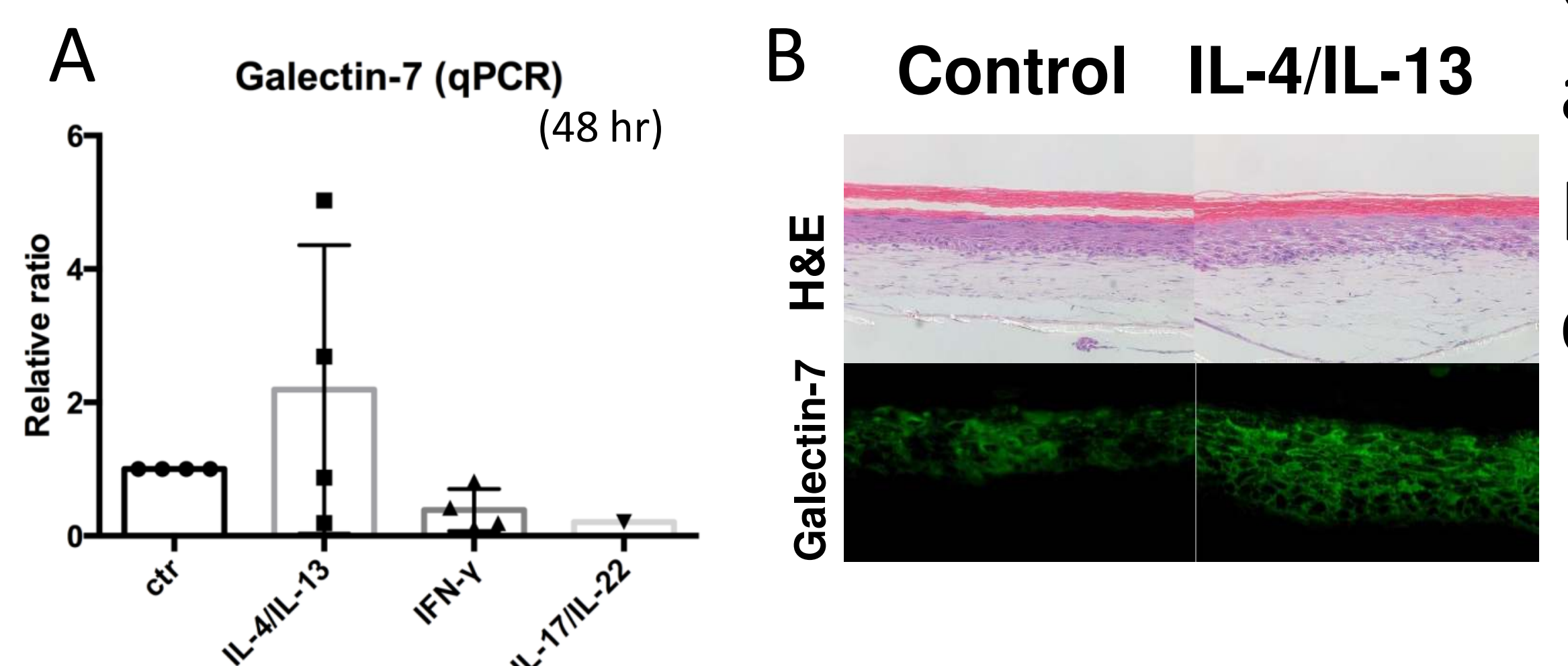

We further investigated the galectin-7 expression in 3D cultured NHEK with or without each cytokines. In consistent with Fig 3, IL-4/IL-13 stimulation facilitated the Gal-7 mRNA expression level/ in 3D cultured skin after $48 \mathrm{hr}(4-\mathrm{A})$.

IL-4/IL-13 induced the spongiosis and slight acanthosis, and promoted the galectin-7 accumulation in the intercellular space or in the cornified layer of keratinocytes, as observed in the lesional skin samples from $A D(4-B)$.

\section{Summary}

- A serum level of Galectin-7 was increased in AD patients and correlated with both disease severity and barrier dysfunction

- Galectin-7 was produced from epidermal keratinocytes and accumulated in the intercellular space of epidermal keratinocytes and stratum corneum - Galectin-7 could be produced extracellularly from keratinocytes via IL-4/IL-13 stimulation

\section{Conclusion}

Galectin-7 expression level in both serum and lesional skin can be a useful biomarker for Th2induced barrier disruption in AD patients 